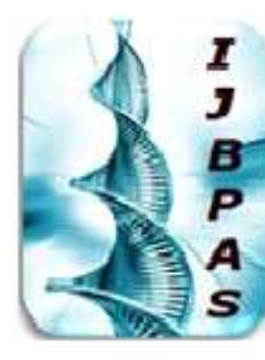

International Journal of Biology, Pharmacy and Allied Seiences (IJBPAS)

'A Bridge Betusen Caboratory and Qpade'

\title{
WwW.ibpas,com
}

\section{In- vivo STUDY OF INDIRUBIN IN PROMASTIGOTES OF L.donovani}

\author{
AHMED MQ ${ }^{1}$, SHAHID SMA ${ }^{2}$, ANWAR $S^{2}$, ACAR T ${ }^{3}$, SIDDIQUIMOHAMMED \\ $\mathrm{AH}^{4}$, FAIYAZ SSM ${ }^{5}$, HASSAN AKB ${ }^{6}$, KAUSAR MA ${ }^{2 *}$ \\ ${ }^{1}$ Department of Pharmacology, College of Medicine, University of Hail, Hail, K.S.A. \\ ${ }^{2}$ Department of Biochemistry, College of Medicine, University of Hail, Hail, K.S.A. \\ ${ }^{3}$ Department of Physical Therapy, College of Applied Medical Science, University of Hail, KSA \\ ${ }^{4}$ Department of Pharmacology, College of pharmacy, Jazan University, K.S.A. \\ ${ }^{5}$ Department of Physiology, College of Medicine, University of Hail, Hail, K.S.A. \\ ${ }^{6}$ Department of Pathology College of Medicine, University of Hail, Hail, K.S.A.
}

*Corresponding Author E Mail: adnankausar1@gmail.com

Received 12 ${ }^{\text {th }}$ Oct. 2018; Revised $11^{\text {th }}$ Nov. 2018; Accepted 22 ${ }^{\text {nd }}$ Nov. 2018; Available online $1^{\text {st }}$ March 2019 https://doi.org/10.31032/IJBPAS/2019/8.3.4664

ABSTRACT

Leishmaniasis is an important complex of protozoal vector-borne diseases that affect both humans and animals. Leishmania donovani (L. donovani) complex consist of member such as L. infantum, L. donovaniand L. chagasi and has considered as an etiological agent for fatal visceral leishmaniasis disease. Recently, data shows that anti-parasitic agents for overcoming the limitations of present-day leishmaniasis chemotherapy toxicity. Indirubin is the active component of Danggui Longhui Wan (a traditional Chinese medicine formulation). Indirubin has been used in herbal medicine since ancient times, with various biological applications. Indirubin is a non- toxic, highly promising natural antioxidants compound having a wide spectrum of biological function. Although some research has been done on the possible medicinal uses, no studies for drug- development have been carried out as yet. Although the crude extract has numerous medicinal uses, clinical application can be also made only after extensive research on its bioactivity, mechanism of action, pharmacotherapeutics and toxicity studies. The leishmanicidal 
activity progressively lessened with decreasing concentration of drug. In MTT assay, O.D. is decreasing with the increasing concentration of indirubin and $50 \%$ growth inhibitory concentration was $30 \mu \mathrm{M}$ of indirubin. About $50 \%$ of parasites were killed at $30 \mu \mathrm{M}$ (indirubin). In-vivo study indirubin against of L.donovani showed maximum reduction $(50 \%)$ in spleen weight of mice. According to the present data, Indirubin showed goodanti- leishmanial activity against promastigotes of $L$. donovanistarin AG83. It is expected the indirubin may find application as a novel drug in the near future to control leishmaniasis.

\section{Keyword: Leishmaniasis, Indirubin, Leishmania donovani, L. macropodum, L. enriettii}

\section{INTRODUCTION}

Leishmaniasis is vector-borne abandoned tropical disease caused by the bite of female phlebotomine sand flies and Trypanosomatid parasitic protozoa coming under genus Leishmania [1]. The Leishmania genus contains two subgenera "Leishmania and Viannia."Leishmania is maincause of Leishmaniasis in human while other organisms Leishmaniasis caused by both subgenera. Mundinia is the third subgenera of Leishmania have proposed for the L. enriettiicomplex, which epidemiologically differs from other Leishmania. The L. enriettiicomplex presently contains $L$. macropodum, $L$. enriettii, formerly known as " $L$. australiensis", L. martiniquensis, and "L. siamensis" formerly proposed names are not yet known and unknown Leishmania isolated from Ghana people. In Thailand, distinct species identified "L. siamensis" from human as well as animal clinical cases amused as L. martiniquensis.

\section{Leishmania promastigote} parasites transmitted the mammals as well as humans through bite of female phlebotomine sand flies [2, 3]. Several million people worldwide infected by parasites $[4,5]$, mainly in the Indian Subcontinentals, Sub-saharan Africa region and Brazil, cause a spectrum of disease ranging from simple, self-healing harmless oriental shore to fatal visceral leishmaniasis or commonly known in India as kala-azar or dumdum.

L. donovani complex consists of the member such as L. donovani, $L$. infantum, and L. chagasi and has considered as anetiological agents for fatal visceral leishmaniasis disease. Sole causative vector for visceral 
leishmaniasisis L. donovani, especially in Indian sub-continent, and it's fatal if it remains not diagnosed. In mammalian blood stream Leishmania promastigotes pass then they are phagocytosed by macrophages and if located in macrophages phagolysosomes, promastigotes converted into non-motile amastigote form with multiple forms [6]. Leishmania infected risk is in more than 350 million people and around 1.5-2 million new cases found with resulted that 500,000 deaths every year in the endemic areas [1]. Resistance increases of Leishmania parasites whereas current therapy toxicity as well as non-existence of a human vaccine which create urgent requirement to discover effective and targeted drugs for the treatment of leishmaniasis [7, 8]. Natural products research leads to be promising for discovering new targeted structures in ranges of diseases including leishmaniasis $[8,9]$.

Throughout natural product scaffolds, alkaloids display extensivestructure diversity can be exploited for the innovation of novel antileishmanial [8, 10]. In addition, marine indole-based alkaloid scaffolds [10] such as variolin [11], roscovitine
[12], leucettines and halogenated indirubins [14], known to target kinases, represent significantly structured compounds for the innovation of new targeted for treatment of antileishmanial $[15,16]$.

An active ingredient Indirubin (Figure-1) is the Chinese traditional medicine "Danggui Longhui Wan", containing plants like Indigo feratinctoria L. and Isatistinctoria L. In 1980s China shows interest in clinical use of indirubin, when scientists with medical doctors together started examined it's as clinically use for the treatment of chronic myelocytic leukemia (CML), overproduction of granulocytes characterized the slowly progressive disease [17-20]. Around $50 \%$ of the treated CML patients exhibited fractional or complete remission [19-22], comparable to the advanced treatment using the cytostatic agent busulfan [21] Toxicity of Indirubin was very low whereas side effects experienced by about half of the members suffer from mild abdominal pain, nausea, and diarrhea [20]. Reversible pulmonary arterial hypertension and cardiac insufficiency were reported in three cases [23]. 


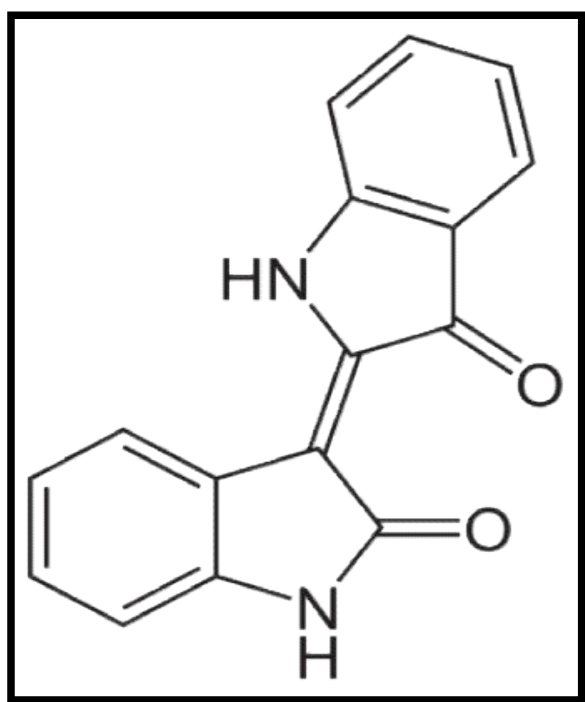

Figure 1: Chemical structure of indirubin

\section{METHOD AND MATERIALS}

\subsection{Parasites:}

Leishmania parasites used in this study are L.donovani (AG83) Indian strain were cultivated in $\mathrm{BALB} / \mathrm{c}$ mice. Promastigotes of this strain were cultivated in medium 199 supplemented with $10 \%$ heat inactivatedfoetal bovine serum, 100 units of penicillin $\mathrm{G} / \mathrm{ml}$ and $100 \mu \mathrm{g}$ of streptomycin sulphate $/ \mathrm{ml}$ at $22^{\circ}$ C.Parasites viability was determined microscopically. The log phase viable promastigotes were further subcultured in M199 at $22^{\circ}$ C.Promastigotes viability wasmonitored throughout the culture period.

\section{2 $\mathrm{BALB} / \mathrm{c}$ mice female}

$\mathrm{BALB} / \mathrm{c}$ female mice around 30 to 35 grams, about 28 to 35 days were used for the study. Animals were maintained at $25^{\circ} \mathrm{C}$ under suitable parameter.

\subsection{Cell Cytotoxicity Assay}

MTT assay is a standard colorimetric assay which measures changes in color for measuring cellular proliferation. It is mainly used for the determination of toxic materials and cytotoxicity of potential medicinal agents [21].

Yellow MTT (3-4, 5dimethylthiazol $\quad-2$-yl)-2, 5 diphenyltetrazolium bromide, a tetrazole) is reduced to purple formazan in the mitochondria of living cells. A solublization solution $(0.4 \mathrm{~N} \mathrm{HCl}$ diluted in isopropanol) is added to dissolve the insoluble purple formazan product into a colored solution. In ELISA reader absorbance of colored solution quantified by measuring at a certain wavelength 
(550 to570nm) and plots the graph with the help of graph pad.

\subsection{In-vivo antileishmanial Activity of indirubin}

Swiss albino mice (28-35 days) were infected in-vivo through freshly transformed promastigotes of L.donovani ( $2 \times 10^{7} /$ mouse) [25]. Doses of drugs indirubin started two months after infection. Indirubin high dose $(1.0 \mathrm{mg} / \mathrm{kg}) /$ bodyweight) was injected to two groups of mice intraperitoneally. Pentamidine used as a positive control, empty served as infected control.

\subsection{Micewere divided into following four} groups which are as follows

1. Infected mice treated with PBS

2. Infected mice treated with Pentamidine control.

3. Infected mice treated with high dose of indirubin $(1.0 \mathrm{mg} / \mathrm{kg}$ body weight).

4. Mice have taken as healthy control in which no drug was administered.

\section{RESULTS}

\subsection{Effect of indirubin in L.} donovanipromastigotes
In order to determine the concentration of indirubin at which approximately 50\% death of $L$. donovani promastigotes would occur, we had tested the effect of several concentration of the drug via the MTT assay. Data shows of $L$. donovani promastigotes under the invitro conditions. The death profile was initially slow when concentration 5-50 $\mu \mathrm{M}$ were used. Subsequently, a very rapid and dose dependent death occurred with indirubin concentration between $30 \mu \mathrm{M}$ shown in Figure 2.

\subsection{In-vivo assay}

The in-vivo leishmanicidal activity of indirubin against promastigotes of L.donovani is shown in figure-3.

In case of spleen weight after 21 days post treatment, maximum reduction $(50 \%)$ in spleen weight was observed in case of indirubin whereas $41 \%$ was observed in case of pentamidine respectively.

After 35 days post treatment, maximum reduction in spleen weight $(55 \%)$ was observed in case of indirubin whereas in case of pentamidine it was $52 \%$ while comparing with infected control. 


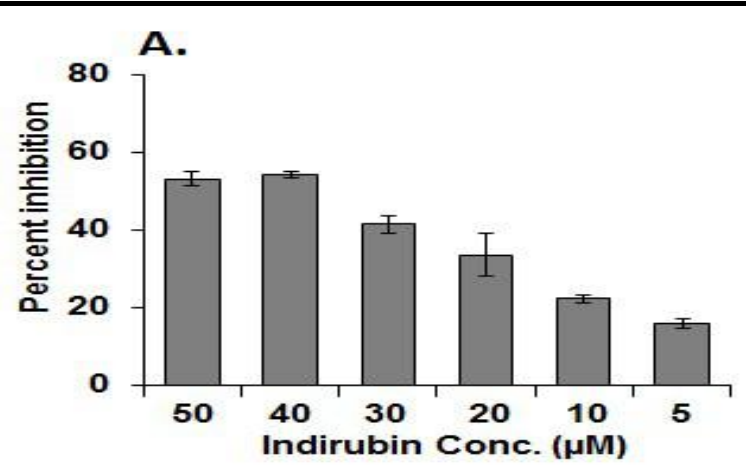

B.

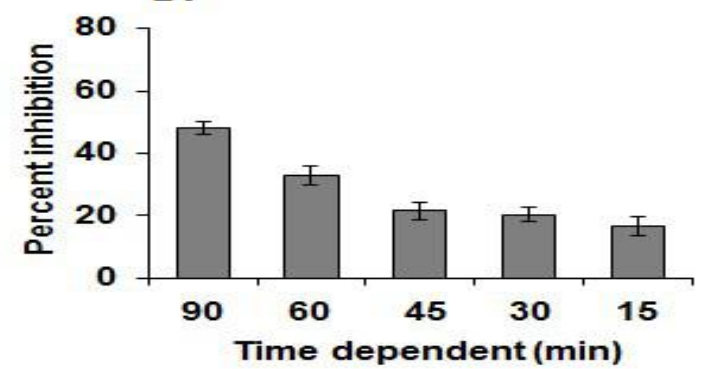

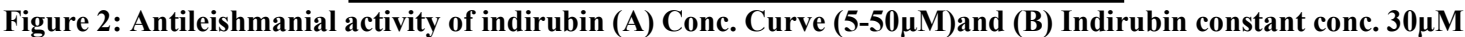
at different time interval (15-90 min)

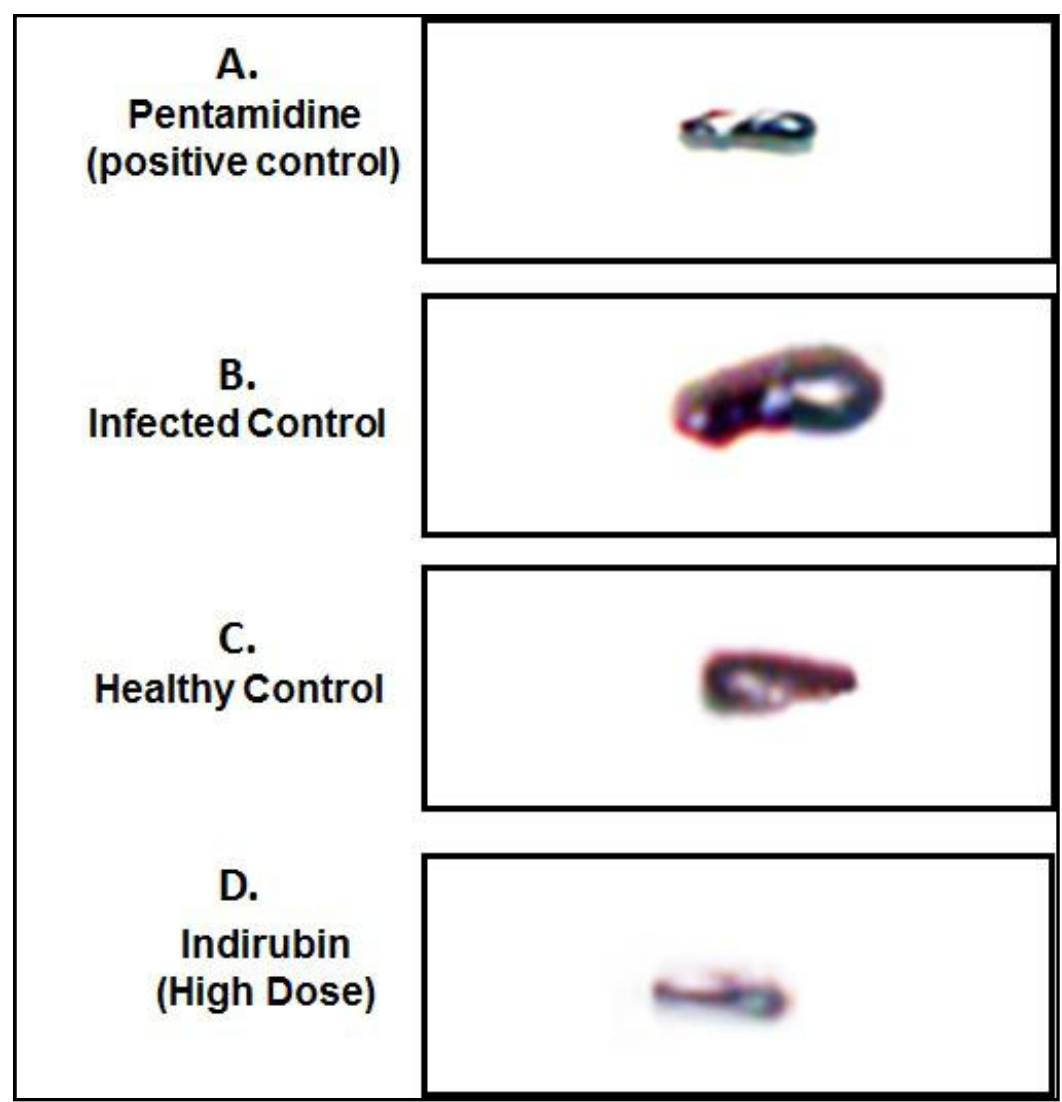

Figure-3: Dramatic reduction in size of the spleen of mice showing significant differences (A) Positive Control, (B) Infected with parasite (C) Healthy control and (D) indirubin treatment 


\section{DISCUSSION}

In the present study, indirubin shows leishmanicidal activity on promastigotes of L.donovani in vitro. It is known that natural products have a future in the search for new and selective agents for the treatment of leishmaniasis [26]. A majoradvantage of indirubin screening is the structural diversity of substances that could create natural products a source of novel lead compounds against leishmaniasis. Indirubin showed about $50 \%$ inhibition at $40 \mu \mathrm{M}$ concentration on promastigotes of Leishmania donovani parasites by MTT assay. All these results encourage furthering investigating the leishmanicidal activity in-vivo with indirubin to check whether indirubinhas promising anti-leishmanial activity invivo and in-vitro.

The antileishmanial activity could be seen with the help of MTT assay. In MTT assay concentration of drug was $5 \mu \mathrm{M}$ $50 \mu \mathrm{M}$. At $50 \mu \mathrm{M}$ concentration, indirubin showed more than $54 \%$ at high dose concentration cell proliferation could be seen.

In case of spleen weight after 21 days post treatment, maximum reduction $(50 \%)$ in spleen weight was observed whereas $41 \%$ and $55 \%$ were observed in case of pentamidine and indirubin respectively.

These in-vitro and in-vivo results clearly suggest that indirubin are a better antileishmanial agent than the standard drug which is frequently used in therapy are toxic but also introduce the targeted delivery system for intracellular parasites. The results presented here provide motivation for further exploration of these compounds, particularly as antileishmanial agents. Laboratory synthesis and the possibility of modifying the chemical structure of indirubin are important advantages for the development of new antileishmanial agents using as herbal plants.

\section{CONCLUSION}

Indirubin used as Herbal medicine since ancient times mainly started used in China, with biological applications. Although in possible medicinal applications some work has been done, but no studies have been carried out yet for development of drug drugdevelopment. On the other hand, the crude extract has numerous medicinal applications, clinical applications confirmed after extensive research on its pharmacological therapeutic, bioactivity, mechanism of action and toxicity studies. 
However, availability of indirubin in pure form which indicates a broad spectrum of biological activities and help to make easily work to develop the new drugs from this compound after extensive studies on its mechanism of action and pharmacological effects. Recently few years ago we have seen an increased frequently interest shownfor treating various diseases with natural products. As studied the characteristics of indirubin are non-toxic, highly promising natural antioxidant compound with broad range of biological function. According to the present data indirubin showed good antileishmanial activity against promastigotes of L.donovani strain (AG83).It is expected that indirubin may find application as a novel drug in the near future to control Leishmaniasis.

Conflict of Interest: The author declares that there is no conflict of interest regarding the publication of this paper.

\section{REFERENCES}

[1] WHO: Control of Leishmaniases, WHO Technical Report Series. ; 2010:949.

[2] Luder CG, Campos-Salinas J, Gonzalez-Rey E, van Zandbergen G: Impact of protozoan cell death on parasite-host interactions and pathogenesis. ParasitVector 3: 2010; 116.

[3] Dostalova A, Volf P: Leishmania development in sand flies: parasite-vector interactions overview. Parasit Vector5: 2012; 276.

[4] Saeed M, Faisal SM, Ahmad I, Kausar MA, Alam MJ, Khan S, Mustafa H. Status of lymphatic filariasis with progression of age and gender \& eradication strategies: A survey among residents of Hardoi district of Uttar Pradesh, an endemic region of North India. Cell Mol Biol (Noisy-le-grand). $\quad 31 ; \quad 2018$, 64(4):46-51.

[5] Saeed, M., Al-Shammari, E.M., Khan, S., Alam, M.J. and Adnan, M. Monitoring and evaluation of lymphatic filariasis interventions: current trends for diagnosis. Reviews in Medical Microbiology, 27(2). 2016, 75-83. [6] Stuart K, Brun R, Croft S, Fairlamb A, Gurtler RE, McKerrow J, Reed S, Tarleton R: Kinetoplastids: related protozoan pathogens, different diseases. J 
Clin. Invest. 118(4): 2008; 13011310.

[7] Croft SL, Sundar S, Fairlamb AH: Drug resistance in leishmaniasis. Clin. Microbiol. Rev. 19(1): 2006; 111-126.

[8] Polonio T, Efferth T: Leishmaniasis: drug resistance and natural products (review). Int J Mol Med 22(3): 2008; 277-286.

[9] de Paula RC, da Silva SM, Faria KF, Frézard F, Moreira CPS, Foubert K, Lopes JCD, Campana PRV, Rocha MP, Silva AF, Silva CG, Pieters L, Almeida VL. In vitro antileishmanial activity of leaf and stem extracts of seven Brazilian plant species. J Ethnopharmacol. 25 (232): 2019; 155-164.

[10] Hussain, M.S., Fareed, S., Saba Ansari, M., Rahman, A., Ahmad, I.Z. and Saeed, M. Current approaches toward production of secondary plant metabolites. Journal of pharmacy \&bioallied sciences, 4(1), 2012, 10-20.

[11] Skropeta D, Pastro N, Zivanovic A: Kinase inhibitors from marine sponges. Mar Drugs, 9(10): 2011; 2131-2154.

[12] Walker SR, Carter EJ, Huff BC, Morris JC: Variolinsand related alkaloids. Chem. Rev. 109(7): 2009; 3080-3098.

[13] Tahtouh T, Elkins JM, Filippakopoulos P, Soundararajan M, Burgy G, Durieu E, Cochet C, Schmid RS, Lo DC, Delhommel F, Oberholzer AE, Pearl LH, Carreaux F, Bazureau JP, Knapp S, Meijer L: Selectivity, cocrystal structures, and neuroprotective properties of leucettines, a family of protein kinase inhibitors derived from the marine sponge alkaloid leucettamine b. J Med Chem 55(21): 2012; 9312-9330.

[14] Meijer L, Skaltsounis AL, Magiatis P, Polychronopoulos P, Knockaert M, Leost M, Ryan XP, Vonica CA, Brivanlou A, Dajani R, Crovace C, Tarricone C, Musacchio A, Roe SM, Pearl L, Greengard P: GSK-3selective inhibitors derived from Tyrian purple indirubins. 
ChemBiol 10(12): 2003; 1255 1266.

[15] Liu J, Hu Y, Waller DL, Wang J, Liu Q: Natural products as kinase inhibitors. Nat Prod Rep, 29(3): 2012; 392-403.

[16] Xingi E, Smirlis D, Myrianthopoulos V, Magiatis $\mathrm{P}$, Grant KM, Meijer L, Mikros E, Skaltsounis AL, Soteriadou K: 6-Br-5methylindirubin-3'oxime (5-Me-6-BIO) targeting the leishmanial glycogen synthase kinase-3 (GSK-3) short form affects cell-cycle progression and induces apoptosis-like death: exploitation of GSK-3 for treating leishmaniasis. Int $\mathrm{J}$ Parasitol, 39(12): 2009; 1289_ 1303.

[17] Clinical and experimental studies in the treatment of chronic granulocytic leukemia with indirubin. Zhonghua Nei Ke Za Zhi,18(2): 1979; 83-88,

[18] Wu GY, Fang FD. Studies on the mechanism of indirubin action in the treatment of chronic granulocytic leukemia. II. Effects of indirubin on nucleic acid and protein synthesis in animal transplantable tumor cells and normal proliferating cells in vitro (author's transl). Zhongguo Yi Xue Ke Xue Yuan Xue Bao. 2(2): 1980; 83-87,

[19] Ma MZ and Yao BY. Progress in indirubin treatment of chronic myelocytic leukemia. Journal of Traditional Chinese Medicine. $3(3), 1983 ; 245-248$.

[20] Xiao Z, Hao Y,Liu B, and Qian $\mathrm{L}$,.Indirubin and meisoindigo in the treatment of chronic myelogenous leukemia in China. Leukemia \& Lymphoma. 43(9): 2002; 1763-1768.

[21] Zhang ZN, Liu EK, Zheng TL, and Li DG, Treatment of chronic myelocytic leukemia (CML) by traditional Chinese medicine and Western medicine alternately," Journal of Traditional Chinese Medicine. 5(4): 1985; 246-248,.

[22] Hoessel R, Leclerc S, Endicott JA et al., Indirubin, the active constituent of a Chinese antileukaemia medicine, inhibits cyclin-dependent kinases. Nature Cell Biology,1(1): 1999; $60-67$, 
[23] Jiang $\mathrm{S}, \mathrm{Yu} \mathrm{G}$ and $\mathrm{Cao} \mathrm{J}$. Adverse effect of indirubin on the cardiovascular system, a report of 3 cases. Chinical Journal of Hematology. 7(1) 1986:30.

[24] Efstathiou A, Gaboriaud-Kolar N, Smirlis D, Myrianthopoulos V, Vougogiannopoulou K, Alexandratos A, Kritsanida M, Mikros E, Soteriadou K, Skaltsounis AL. An inhibitordriven study for enhancing the selectivity of indirubin derivatives towards leishmanial Glycogen Synthase Kinase-3 over leishmanial cdc2-related protein kinase 3. Parasit Vectors. 2014 May 20; 7: 234.

[25] Xingi E, Smirlis D, Myrianthopoulos V, Magiatis P, Grant KM, Meijer L, Mikros E, Skaltsounis AL, Soteriadou K. 6-Br-5methylindirubin-3'oxime (5-Me-6-BIO) targeting the leishmanial glycogen synthase kinase-3 (GSK-3) short form affects cell-cycle progression and induces apoptosis-like death: exploitation of GSK-3 for treating leishmaniasis. Int $\mathbf{J}$
Parasitol. 2009 Oct;39(12):1289303.

[26] Rudrapaul P, Sarma IS, Das N, De UC, Bhattacharjee S, Dinda B. New flavonol methyl ether from the leaves of Vitex peduncularis exhibits potential inhibitory activity against Leishmania donovani through activation of iNOS expression. Eur J Med Chem. 2014; 87:32835. 